\title{
L'intégration Des TIC Dans L'enseignement Des Sciences De La Vie Et De La Terre Au Maroc: Etat Des Lieux Et Défis À Relever
}

\author{
Youssef Nafidi \\ Formation Doctorale en Didactique des Sciences et Ingénierie Pédagogique, \\ Centre d'Etudes Doctorales « Sciences et Techniques », LIRDIST, \\ Faculté des Sciences Dhar Mahraz, \\ Université Sidi Mohammed Ben Abdellah, Fès-Maroc

\section{Anouar Alami} \\ Professeur de Chimie Bio-Organique, Laboratoire de Chimie Organique \\ (LCO), Laboratoire Interdisciplinaire de Recherche en Didactique des \\ Sciences et Techniques, LIRDIST, Faculté des Sciences Dhar Mahraz, \\ Université Sidi Mohammed Ben Abdellah, Fès-Maroc

\section{Moncef Zaki} \\ Laboratoire Interdisciplinaire de Recherche en Didactique des Sciences et \\ Techniques, Faculté des Sciences Dhar Mahraz, \\ Université Sidi Mohammed Ben Abdellah, Fès-Maroc
}

\section{Bouchta El Batri}

Formation Doctorale en Didactique des Sciences et Ingénierie Pédagogique,

Centre d'Etudes Doctorales « Sciences et Techniques », LIRDIST,

Faculté des Sciences Dhar Mahraz,

Université Sidi Mohammed Ben Abdellah, Fès-Maroc

Mohammed Elazami Hassani

Hanane Afkar

Centre Régional des Métiers d'Education

et de Formation de Fès-Meknès, Maroc

Doi: 10.19044/esj.2018.v14n1p97 URL:http://dx.doi.org/10.19044/esj.2018.v14n1p97

\begin{abstract}
Information and Communication Technologies (ICT) are important educational tools; the measures taken to integrate them into the Moroccan education system are multiple. This study was conducted among 181 teachers of life and earth sciences in a large number of establishments (colleges and high schools). This paper focuses on presenting a diagnosis of the reality of the use of ICT in the teaching of the life sciences and earth in secondary school at the national level; identify teachers' perceptions of the real benefits offered
\end{abstract}


by the pedagogical integration of these means in education; and determine, according to the conception of the teachers themselves, the main factors that impede or facilitate the effective and efficient integration of ICT in teaching practice. The results of this research show that there is a limited use of ICT, even though most teachers interviewed are convinced of the richness of pedagogical opportunities created by the integration of ICT in education. Thus, ICT generalization projects in the Moroccan education system should take the main constraints highlighted in this research to integrate the digital age into our schools on a more solid foundation.

Keywords: ICT, life and earth sciences, integration, obstacles, teaching

\section{Résumé}

Les Technologies d'Information et de Communication (TIC) sont des outils éducatifs incontournables, les mesures entreprises pour les intégrer dans le système éducatif marocain sont multiples. Cette étude menée auprès de 181 enseignants des Sciences de la Vie et de la Terre (SVT) répartis sur un nombre important des établissements (collèges et lycées), vise à présenter un diagnostic de la réalité de l'usage des TIC dans l'enseignement des SVT au secondaire à l'échelle nationale, à identifier les perceptions des enseignants des SVT des bénéfices véritablement apportés par l'intégration pédagogique de ces moyens dans l'éducation et également à déterminer, selon la conception des enseignants eux-mêmes, les principaux facteurs qui entravent ou facilitent une intégration effective et efficiente des TIC dans la pratique enseignante. L'ensemble des résultats de cette recherche permet de retenir qu'il y a une utilisation limitée malgré que la majorité des enseignants interrogés sont convaincus de la richesse des opportunités pédagogiques induites par l'intégration des TIC dans le travail enseignant. Ainsi, les projets de généralisation des TIC dans le système éducatif marocain devraient prendre en considération les principales contraintes soulignées dans cette recherche afin d'intégrer sur des bases plus solides l'ère numérique dans nos établissements scolaires.

Mots-clefs: TIC, Sciences de la Vie et de la Terre, intégration, obstacles, enseignement

\section{Introduction}

$\mathrm{Au}$ cours des dernières années, les nouvelles Technologies de l'Information de la Communication (TIC) évoluent rapidement et sont introduites dans les différents domaines de notre société et affectent, de façon remarquable, les dimensions économiques, sociales, politiques, culturelles et éducatives (Traoré, 2008; Redecker et al., 2009; Biaz et al., 2009; Maddux et 
al., 2012; Collin, et al., 2013; Ait Kaikai, 2014). Ces technologies, lorsqu'elles sont combinées et interconnectées, permettent de rechercher, de stocker, de traiter et de diffuser une quantité impressionnante d'informations, sous forme de données de divers types (texte, son, images, simulations, etc.), et peuvent être utilisées pour échanger, communiquer, collaborer, coopérer, produire, créer et publier (Basque et al., 2003; Mastafi, 2013).

L'intégration des TIC apporte plusieurs bénéfices tels que la flexibilité, l'accessibilité, l'accroissement des échanges et interactions entre les divers acteurs (Karsenti, 2003; Nafidi et al., 2015). Les TIC délocalisent, dans le temps et dans l'espace, les échanges entre les enseignants et les apprenants, et diversifient de ce fait les activités d'apprentissage et les modes d'enseignement et d'apprentissage (Peraya, 2005; Depover et al., 2007).

Le domaine de l'éducation doit profiter absolument de l'apport de ces technologies et doit être conscient que ces TIC fournissent des moyens novateurs, non seulement pour la diffusion d'une quantité prodigieuse des connaissances, mais également pour envisager de nouvelles directions pour enrichir, améliorer, voire transformer certaines pratiques éducatives. Cependant l'intégration réussie des TIC dans l'enseignement, nécessite que les enseignants soient bien outillés de méthodes nécessaires pour une utilisation efficiente de ces technologies dans le processus enseignement-apprentissage (Ahaji, 2012).

L'usage des TIC à des fins d'enseignement et d'apprentissage est approprié aux pédagogies «actives » dans le sens où Freinet l'entendait, il peut encourager un apprentissage actif et contribuer au développement de certaines compétences transversales des apprenants telles que l'organisation, le traitement, l'exploitation, la production et le partage de l'information (Biaz et al., 2009). De ce fait, il peut contribuer à une amélioration de la pédagogie et du rapport au savoir (Perrenoud, 1998; Peraya, 2002; Peraya et al., 2002; Heer et al., 2006).

L'utilisation des Technologies d'Information et de Communication dans l'Enseignement (TICE) en SVT a fortement multiplié les possibilités pédagogiques permettant d'aborder les concepts de cette .discipline scientifique. On citera par exemple les possibilités qu'offrent les TIC dans certaines situations d'apprentissage : voir en modèle réduit ou en accéléré des événements qui ne sont pas accessibles à l'observation directe pour des raisons de temps (par exemple vitesse très faible de certains phénomènes géologiques ou biologiques) ou d'espace (dimensions gigantesques ou microscopiques de certains objets géologiques), le traitement de données permettant de construire ou d'enrichir des concepts scientifiques (exemple utilisation des logiciels de comparaison de séquences nucléiques) et la simulation des phénomènes et des mécanismes biologiques ou géologiques. 
Le Maroc, convaincu des apports et des potentialités offertes par l'innovation technologique, mène de nombreuses initiatives visant la mise en place de programmes de généralisation et d'introduction des TIC dans le système éducatif afin d'améliorer la qualité de l'enseignement et de la formation, l'adapter aux standards internationaux en vigueur et en faire un vecteur de développement social et économique. Les actions ambitieuses et les projets intégrés adoptés par les décideurs en matière d'intégration des TIC dans l'apprentissage et la formation sont nombreux:

Le Réseau MARWAN (Maroc Wide Area Network initié en 1998) : Un réseau informatique national dédié à l'éducation et la recherche scientifique. Dans sa nouvelle version, MARWAN 3 (2009-2012) offre aux universités marocaines l'accès à internet haut débit grâce à sa connexion, avec un réseau, réservée particulièrement au trafic académique.

La Charte Nationale D'éducation et de Formation (1999): La stratégie de promouvoir l'intégration des TIC dans l'enseignement au Maroc est soulignée dans le levier 10 : «Considérant que la technologie pédagogique joue un rôle déterminant et croissant dans les systèmes et méthodes d'enseignement..., les autorités d'éducation et de formation veilleront à intégrer ces technologies dans la réalité de l'école, sur la base de l'objectif suivant : un centre informatique et une bibliothèque multimédia dans chaque établissement au terme de la décennie prochaine à partir de la rentrée scolaire 2000-2001».

Le Campus Virtuel Marocain (2004): Dans le cadre de ce projet, des Centres de Ressources Universitaires (CRU) sont créés dans toutes les universités marocaines en vue de soutenir l'insertion des TIC dans l'enseignement universitaire. La mission principale des CRU est de former et accompagner les enseignants, et mettre à leur disposition les outils technologiques nécessaires à l'intégration des TIC dans les pratiques éducatives (plateformes, applications éducatives, etc.).

Le Concours des Enseignants Innovants (2005): Le fruit de la coopération entre Microsoft et le ministère de l'éducation nationale dans le cadre de l'initiative "Partenaires du Savoir ». Il avait pour objectif de contribuer à diffuser et à valoriser l'innovation pédagogique dans les établissements d'enseignement, créer un dynamisme et une compétitivité entre les acteurs pédagogiques et élargir l'accès actif à la technologie et améliorer son utilisation dans le processus de l'enseignement-apprentissage (Nachit, 2013).

Le Programme GENIE (lancé en 2006 et révisé en 2009): Ce programme, adopté par le gouvernement en mars 2005, est la dimension opérationnelle de la stratégie nationale de généralisation des TIC dans l'éducation. Il se décline en quatre axes convergents: 
$\checkmark \quad$ Infrastructure: Doter les écoles de la connexion internet, fournir l'infrastructure informatique pour équiper les établissements scolaires de salles informatiques et de valises multimédias (VMM).

$\checkmark \quad$ Formation des Enseignants et Cadres Administratifs: Former environ 200.000 personnes (directeurs d'établissement, inspecteurs et enseignants) à l'informatique et à l'utilisation des TIC dans l'environnement pédagogique des élèves.

$\checkmark \quad$ Ressources Numériques: Créer un laboratoire national des ressources numériques ainsi qu'un portail national TICE pour accélérer le développement des contenus numériques et doter tous les établissements scolaires de ressources numériques nécessaires.

$\checkmark \quad$ Développement des Usages: Cet axe est une nouveauté introduite suite à la révision de la stratégie du programme GENIE en 2009. Son objectif fondamental est d'assurer un bon accompagnement aux usagers, la communication, la sensibilisation et le partage de bonnes pratiques.

Le Génie Sup (2008): Un programme de généralisation des technologies d'information et de communication dans l'enseignement supérieur. Il vise le développement de la culture numérique de tous les acteurs de l'université (étudiants, enseignants, personnel administratif) et promouvoir l'intégration des TIC dans l'enseignement, la recherche et la gouvernance des établissements de l'enseignement supérieur.

- $\quad$ Le Projet Nafida (2008): La Fondation Mohammed VI de promotion des œuvres sociales de l'éducation-formation a lancé le programme Nafida, en collaboration avec le ministère de l'éducation nationale, pour faciliter l'accès de la famille de l'enseignement aux technologies de l'information et de la communication. Nafida avait pour objectif initial de connecter 100.000 enseignants adhérents à l'internet (mobile ou fixe) et d'aider chaque adhérent à acquérir un ordinateur portable, équipé d'un système d'exploitation Windows et de la suite bureautique MS (Office Standard), en proposant une large gamme de produits de grande qualité à des tarifs préférentiels, négociés et subventionnés, à hauteur de $2.000 \mathrm{DH}$ par bénéficiaire.

L’opération Injaz (2009): Programme lancé dans le cadre du Maroc numérique 2013, consiste à subventionner les frais d'achat des ordinateurs portables et d'abonnement à internet à hauteur de $70 \%$ au profit des élèves ingénieurs, des étudiants inscrits au Master et aux centres doctoraux.

- $\quad$ Le Portail National Taalimtice (2011): Un site très intéressant, a été créé et mis en ligne, permet à tous les acteurs du système éducatif marocain de s'informer sur les TICE, l'échange des informations, la participation active au développement et à la diffusion de ressources numériques pédagogiques.

- $\quad$ Le Projet Varen (2013): Un projet lancé, par le laboratoire national des ressources numériques, pour promouvoir la production des applications éducatives numériques adaptées au curriculum marocain, par l'octroi d'une 
attestation de validation des ressources éducatives numériques. La labellisation des ressources pédagogiques produites par les enseignants marocains, permet de s'assurer de la conformité du contenu numérique aux programmes nationaux, contrôler la fiabilité scientifique du contenu et garantir la bonne qualité pédagogique et didactique des produits.

Le Plan Maroc Numeric 2013: Ce plan, lancé en 2009 sous la présidence de sa majesté le roi Mohammed VI dans le cadre d'une stratégie nationale pour la société de l'information et de l'économie numérique, a pour ambition de positionner notre pays comme un hub technologique régional et de faire du secteur des TIC un des piliers de l'économie et un vecteur du développement humain.

Programme LAWHATI (2015): Ce programme, initié et piloté par le Ministère de l'Enseignement Supérieur, de la Recherche Scientifique et de la Formation des Cadres dans le but de s'aligner sur les standards internationaux en matière d'intégration et d'utilisation des TIC dans les domaines de l'enseignement, consiste à mettre à la disposition des étudiants inscrits dans les établissements d'enseignement supérieur, des stagiaires inscrits dans les établissements de formation professionnelle, du corps professoral œuvrant dans les établissements universitaires et des formateurs de la formation professionnelle, des Tablettes munies d'un contenu pédagogique intégré.

Le Plan « Maroc Digital 2020 »: Il prendra le relais du plan « Maroc Numeric 2013 » et permettra d'inscrire le pays dans l'économie digitale et participera activement à la réduction de la fracture numérique grâce notamment à la réalisation de $50 \%$ des démarches administratives par internet et connecter $20 \%$ des petites et moyennes entreprises marocaines.

- La Vision Stratégique Pour la Réforme de L'école (2015/2030): L'intégration des TIC dans le système éducatif marocain a figuré parmi les mesures et les projets prioritaires de la mise en œuvre de la réforme éducative de la vision stratégique pour une école de l'équité, de la qualité, et de la promotion (CSEFRS, 2015). Dans le but de renforcer l'intégration des technologies éducatives pour améliorer la qualité des apprentissages et pour favoriser le passage d'une société de consommation du savoir à une société qui le produit et le diffuse, le Conseil Supérieur de l'Education, de la Formation et de la Recherche Scientifique (CSEFRS) recommande de:

$\checkmark \quad$ Elaborer un programme national, visant à compléter l'équipement des établissements scolaires en technologies éducatives, en salles multimédias et en équipement audiovisuel, à les connecter au réseau internet et à équiper les bibliothèques scolaires et les structures universitaires de recherche, en ressources numériques nécessaires.

$\checkmark \quad$ La création des centres de ressources numériques et des laboratoires d'innovation et de production de ressources pédagogiques, ainsi que la formation des spécialistes dans ce domaine. 
$\checkmark \quad$ L’usage pédagogique des logiciels éducatifs et des ressources numériques interactives ciblant l'auto-apprentissage, la recherche, la diversification des sources d'apprentissage, ainsi que la formation des spécialistes pour la conception et le développement de ces outils technologiques.

$\checkmark \quad$ L'Intégration à moyen terme, des TIC comme matière principale dans la formation initiale et continue de tous les acteurs pédagogiques.

$\checkmark \quad$ Le développement et la promotion de la formation à distance comme complément aux cours en présentiel.

$\checkmark \quad$ L'élaboration d'un plan d'action pour la sensibilisation à l'importance de l'introduction des technologies de l'information et de la communication et à leur contribution à la réforme de l'école.

En définitive, le Maroc a adopté de grandes stratégies afin de rattraper les retards en termes d'infrastructures, de formation, de ressources numériques et de développement des usages des TIC. Selon la directrice du programme GENIE : $85 \%$ des établissements scolaires sont actuellement équipés d'un environnement multimédia de base, 266000 du corps pédagogique ont été formés dans le domaine d'utilisation des TICE et 80\% des ressources numériques conformes aux programmes scolaires sont acquises (Laaziz, 2016).

\section{Problématique}

Les enseignants sont sollicités de toutes parts pour intégrer les TIC dans leur pratique pédagogique. L'impact positif de l'usage des technologies éducatives sur le processus d'enseignement et d'apprentissage est mis en relief par un bon nombre de recherches menées à l'échelle internationale (Karsenti et al., 2001; Perreault, 2003; Saunders et al., 2003; Rogers, 2004; Forget, 2005; Depover et al., 2007; Monsakul, 2008; Poyet et al., 2009; Huot et al., 2009; Turney et al., 2009; Szabo et al., 2009; Raby et al., 2011; Boticki et al., 2015; Yang et al., 2015) et aussi bien à l'échelle nationale (Ahaji et al., 2008; El Ouidadi et al., 2011; Lakdim, 2012; Droui et al., 2013; El hassouny et al., 2014; Ouazzani, 2014; Nafidi et al., 2015; Benfares et al., 2015; Alj et al., 2016; Guennoun et al., 2016; Ou-Zennou et al., 2016).

Certes, l'état marocain a alloué ces dernières années un budget important en vue de la généralisation des TIC dans l'enseignement. Selon le responsable du programme national GENIE, 2838 établissements sont équipés en salle multimédia et valise multimédia et connectés à l'Internet avec filtrage, $100 \%$ des enseignants ont été formés pour intégrer les TIC (Laaziz, 2016). Selon l'enquête annuelle de collecte des indicateurs TIC auprès des ménages et des individus qui a été réalisée en 2016 par Association Nationale de la Recherche et de la Technologie (ANRT), le parc des smartphones est estimé à plus de 18 millions, le nombre d'internautes est presque 18,5 millions, $44 \%$ 
des internautes passent entre 1 à 2 heures par jour sur Internet (ANRT, 2017). Les implications engendrées par les défis de la société de l'information d'une part, et les réformes que connaît le système éducatif marocain d'autre part, sont certainement en faveur de la pénétration des TIC dans l'acte pédagogique.

Toutefois, les observations que nous avons faites sur le terrain marocain et les résultats des travaux de recherches menées dans le contexte marocain indiquent que les TIC sont peu utilisées à des fins pédagogiques par les enseignants (Abouhanifa et al., 2008; Biaz et al., 2009; Mastafi, 2013; Alj et al., 2013; El Ouidadi et al., 2013; El Madhi et al., 2014; Maouni et al., 2014; Benfares et al., 2016; Abouzaid et al., 2017). Pour cela, il s'avère très important de s'interroger sur cette situation de faible utilisation des TIC malgré la forte volonté institutionnelle de généraliser l'utilisation de ces technologies dans l'enseignement marocain.

Nous nous intéressons à l'intégration des TIC dans l'enseignement des SVT car il y a encore un manque très significatif des recherches rigoureuses menées pour mieux comprendre la réalité de l'usage des TIC dans l'enseignement de cette discipline au Maroc, notre recherche ne sera pas la première, mais notre approche diffère des précédentes, par sa méthodologie de collecte des réponses des enseignants appartenant à un nombre important de collèges et lycées dispersés dans tout le territoire du royaume. En effet, notre problématique s'énonce par les questions suivantes:

- Quelle est la réalité de l'usage pédagogique des TIC dans l'enseignement secondaire ? Les enseignants font-ils usage des TIC à des fins pédagogiques?

- Quelles sont les perceptions des enseignants quant aux bénéfices tirés de l'intégration pédagogique des TIC en sciences de la vie et de la terre ?

- Quels sont les obstacles d'une intégration effective et efficiente des TIC dans le processus d'enseignement-apprentissage ?

- Quelles sont les conditions d'une intégration réussie des TIC au secondaire?

\section{Méthodologie}

Pour répondre à notre problématique, et évaluer les attitudes des enseignants vis-à-vis de l'intégration des technologies d'information et de communication en sciences de la vie et de la terre au secondaire, nous avons donc procédé à une recherche exploratoire, à travers un questionnaire, que nous avons administré auprès d'un échantillon de convenance (Fortin at al., 2006), obtenant au final 181 répondants.

Le questionnaire a été élaboré et mis en ligne via google Forms, la population d'étude a été sollicitée pour remplir les questionnaires par courriers électroniques et par les réseaux sociaux, principalement Facebook, via les groupes des enseignants des SVT. Cette méthode a été choisie du fait qu'elle 
permet de toucher une large population dispersée sur le territoire géographique.

Le questionnaire, composé de 15 items (fermés et semi-fermés), a été établi autour des points suivants:

- $\quad$ Les données personnelles et l'expérience professionnelle.

- $\quad$ Les équipements institutionnels en matériel informatique.

- $\quad$ La formation et la maitrise des TIC.

- $\quad$ L'usage des TIC dans l'enseignement.

- Les perceptions des enseignants des bénéfices tirés de l'intégration pédagogique des TIC en sciences de la vie et de la terre.

- $\quad$ Les obstacles à l'intégration des TIC en classe.

- $\quad$ Les conditions d'intégration des TIC.

Après le recueil des réponses des enseignants des SVT représentant des groupes d'âge différents, ayant des expériences professionnelles et des compétences d'utilisation des TICE différentes et répartis sur l'ensemble des académies régionales d'éducation et de formation du Maroc. Le traitement pour l'analyse des données a été réalisé à l'aide du logiciel sphinx : ce dernier a été utilisé essentiellement pour le traitement descriptif des répartitions des modalités des différents items du questionnaire.

\section{Résultats et Analyses}

\section{Les Données Personnelles et L'expérience Professionnelle}

Notre échantillon est composé de 181 enseignants de la discipline SVT ( $55,2 \%$ hommes et $44,8 \%$ femmes) ; on peut donc estimer que l'échantillon est relativement équilibré du point de vue du genre.

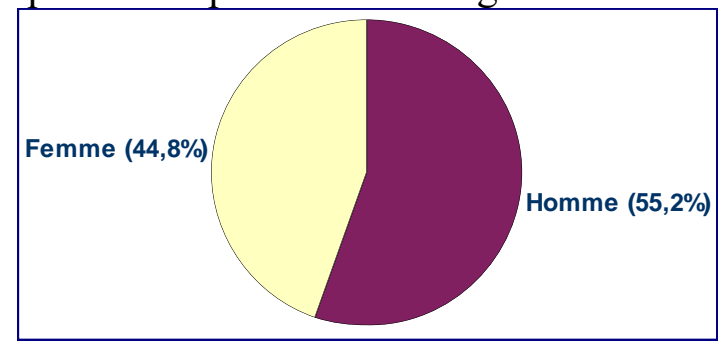

Figure 1. Répartition des répondants en fonction du sexe

D'autre part, l'échantillon de notre étude est formé majoritairement d'enseignants jeunes : 59,66\% des répondants ont une moyenne d'âge inférieure à 30 ans, 27,07\% ont une moyenne d'âge située entre 31 et 40 ans et seulement environ 5\% ont un âge supérieur à 50 ans. La figure ci-dessous montre le profil des âges. 


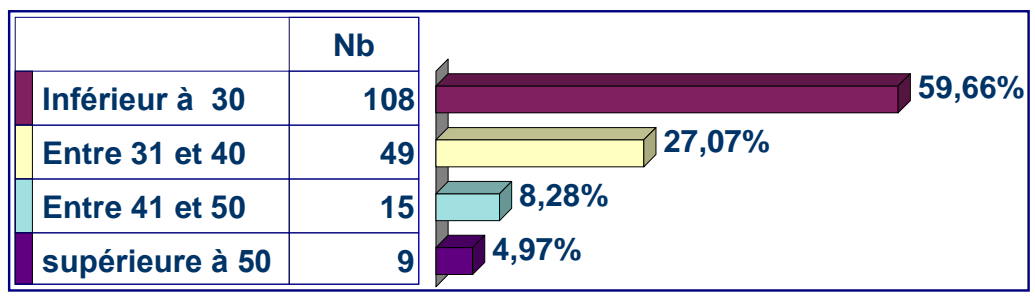

Figure 2. Répartition des répondants en fonction de l'âge

En ce qui concerne l'expérience dans l'enseignement des personnes qui ont répondu à notre questionnaire, $64,6 \%$ ont moins de 5 ans d'ancienneté et seulement $7,7 \%$ ont plus de 20 ans d'ancienneté, ce qui montre bien que cet échantillon se distingue aux échantillons des recherches précédentes. Ce résultat peut être expliqué par le fait qu'on a utilisé un questionnaire électronique et que la vieille communauté d'enseignants est moins familiarisée avec l'utilisation des nouvelles technologies.

\begin{tabular}{|l|r|r|}
\hline & \multicolumn{1}{|c|}{$\mathrm{Nb}$} & \multicolumn{1}{|c|}{$64,6 \%$} \\
\hline Moins de 5 ans & 117 & \\
\hline De 5 à 10 ans & 34 & $18,8 \%$ \\
\hline De 11 à 20 ans & 16 & $8,8 \%$ \\
\hline Supérieur à 20 ans & 14 & $7,7 \%$ \\
\hline
\end{tabular}

Figure 3. Répartition des répondants en fonction de l'ancienneté dans l'enseignement

Par rapport aux cycles d'enseignement relevés dans l'échantillon : $63 \%$ des professeurs interrogés travaillent dans les lycées alors que 37\% travaillent dans les collèges. Ces enseignants sont répartis sur un nombre important des établissements dispersés dans toutes les académies régionales d'éducation et de formation au Maroc. La dispersion de la population cible, qui est due à la mise en ligne de notre questionnaire, est certainement en faveur de mieux connaitre la réalité de l'usage des TIC dans l'enseignement au secondaire.

\begin{tabular}{|c|c|c|}
\hline & $\mathbf{N b}$ & \\
\hline Tanger-Tétouan-Al Hoceïma & 23 & $12,7 \%$ \\
\hline L'Oriental & 21 & $11,6 \%$ \\
\hline Fès-Meknès & 42 & $23,2 \%$ \\
\hline Rabat-Salé-Kénitra & 8 & $4,4 \%$ \\
\hline Béni Mellal-Khénifra & 19 & $10,5 \%$ \\
\hline Casablanca-Settat & 18 & $9,9 \%$ \\
\hline Marrakech-Safi & 15 & $8,3 \%$ \\
\hline Drâa-Tafilalet & 13 & $7,2 \%$ \\
\hline Souss-Massa & 10 & $5,5 \%$ \\
\hline Guelmim-Oued Noun & 6 & $3 \%$ \\
\hline Laâyoune-Sakia El Hamra & 2 & $1,1 \%$ \\
\hline Dakhla-Oued Ed Dahab & 4 & \\
\hline
\end{tabular}

Figure 4. Répartition des répondants en fonction du lieu de travail 


\section{Les Équipements Institutionnels en Matériel Informatique}

D'après les résultats statistiques représentés sur la Figure 5, les sondés déclarent que leurs salles des sciences de la vie et de la terre sont généralement équipées par un ordinateur $(64,6 \%)$ et un vidéoprojecteur (79.6\%). En revanche, très peu de salles sont équipées d'un tableau blanc interactif et de la connexion internet. Par ailleurs, 13,3\% des répondants ont signalé l'absence totale des équipements informatiques mis à leur disposition. Ces résultats montrent clairement que notre pays n'a pas encore pu rattraper les retards en termes d'équipements malgré tous les efforts déployés dans ce domaine.

\begin{tabular}{|l|r|}
\hline & $\mathrm{Nb}$ \\
\hline Ordinateur & 117 \\
\hline Vidéoprojecteur & 144 \\
\hline Tableau blanc interactif & 12 \\
\hline Connexion internet & 20 \\
\hline Aucun matériel informatique & 24 \\
\hline
\end{tabular}

Figure 5. Les équipements institutionnels en outils informatiques

\section{La Formation et la Maitrise des TIC}

D'après les résultats de la Figure 6, plus de la moitié des enseignants des SVT ont acquis le savoir informatique par formation initiale et autoformation, un faible pourcentage des enseignants ont bénéficié de la formation continue et un très faible pourcentage des répondants n'a jamais bénéficié d'une formation concernant l'usage des TIC dans l'enseignement. Ces résultats montrent que la majorité des jeunes enseignants est formée lors de la formation initiale, car un module concernant les TIC est introduit dans le dispositif de formation depuis 2012, une autre catégorie des répondants est intéressée par l'autoformation pour se former et/ou compléter la formation initiale ou continue relative à l'usage des TIC. Toutefois, le nombre des bénéficiaires de la formation continue reste encore très limité (environ 21\%) malgré les efforts déployés, ce qui implique une révision entière de la stratégie adoptée par le ministère d'éducation dans le cadre la formation continue des acteurs pédagogiques (Mastafi, 2014).

\begin{tabular}{|l|r|l|}
\hline & \multicolumn{1}{|c|}{$\mathrm{Nb}$} & \multicolumn{1}{|c|}{$13,8 \%$} \\
\hline Jamais & 25 & \\
\hline Oui, durant la formation initiale & 95 & \\
\hline Oui, durant la formation continue & 38 & \\
\hline Oui, par autoformation & 97 & \\
\hline
\end{tabular}

Figure 6. La formation dans les TIC

D'autre part, le niveau de maitrise des TIC (Logiciels de présentation, navigation sur internet, TBI, EXAO, traitement d'images, usage des 
ressources numériques...) par les enseignants touchés par notre enquête est généralement bon. En effet, seulement 24,3\% ont déclaré que leur niveau de maitrise des TIC est fable à plutôt faible, ceci est expliqué par le faible pourcentage des enseignants qui ne sont pas formés à l'usage des TIC (juste $13,8 \%$ ). Ce résultat positif représente en soi un atout majeur en faveur de l'usage des TIC et encourage même l'utilisation des outils technologiques.

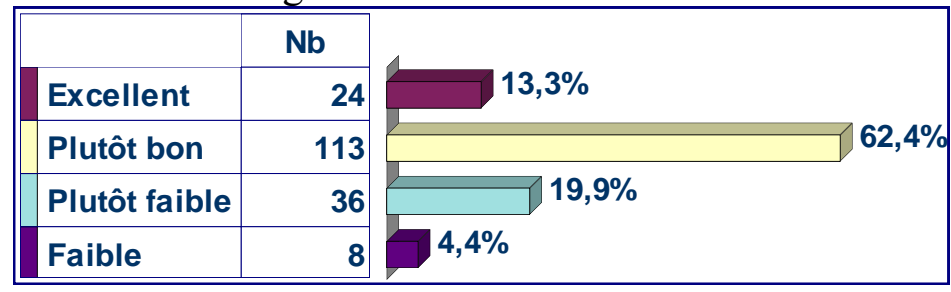

Figure 7. La maitrise des TIC

\section{L'usage des TIC dans L'enseignement}

$40,3 \%$ des enseignants répondants à l'enquête utilisent souvent les TIC dans l'enseignement et presque le même nombre des répondants exploite ces technologies occasionnellement. Alors que moins de $4 \%$ des enseignants de notre échantillon ont déclaré qu'ils n'ont jamais utilisé les TIC en classe. Ainsi, même si nous constatons l'existence d'une proportion importante des enseignants déclarant utiliser régulièrement les TIC, ce résultat ne doit pas être généralisé, car notre échantillon est caractérisé par la prédominance des jeunes enseignants ayant bénéficié de la formation initiale. Il nous semble qu'il y a encore une utilisation limitée des TIC dans l'enseignement des SVT, ce constat est en accord avec les résultats obtenus des enquêtes précédentes (El Madhi et al., 2014; Maouni et al., 2014; Benfares et al., 2016).

\begin{tabular}{|l|r|}
\hline & \multicolumn{2}{|c|}{$\mathrm{Nb}$} \\
\hline Jamais & 6 \\
\hline Rarement & $33,3 \%$ \\
\hline Occasionnellement & 69 \\
\hline Souvent & 73 \\
\hline
\end{tabular}

Figure 8. Utilisation des TIC dans l'enseignement en classe

En ce qui concerne le degré d'utilisation des outils et matériels informatiques (cf. Tableau 1 ci-dessous), il s'avère que la disponibilité des équipements joue un facteur limitant dans l'intégration des TIC en classe. Le vidéoprojecteur et les ressources numériques (animations et simulations) sont suffisamment exploités en salle de cours des SVT du fait de leur disponibilité. En revanche, le tableau blanc interactif, la connexion internet et l'EXAO sont rarement utilisés dans le cadre d'activités pédagogiques à cause du déficit des matériels dans les établissements du secondaire. 
Tableau 1. Degré d'utilisation en salle de cours des outils et matériels informatiques

\begin{tabular}{|c|c|c|c|c|}
\hline & Jamais & Rarement & Occasionnellement & Souvent \\
\hline Vidéoprojecteur & $8,3 \%$ & $19,3 \%$ & $22,7 \%$ & $49,7 \%$ \\
\hline Tableau blanc interactif & $90,1 \%$ & $2,2 \%$ & $3,3 \%$ & $4,4 \%$ \\
\hline Connexion internet & $72,9 \%$ & $11,6 \%$ & $9,9 \%$ & $5,5 \%$ \\
\hline EXAO & $81,7 \%$ & $13,3 \%$ & $4,4 \%$ & $0,6 \%$ \\
\hline Ressources numériques & $6,6 \%$ & $27,6 \%$ & $27,1 \%$ & $38,7 \%$ \\
\hline
\end{tabular}

D'autre part, la Figure 9 révèle clairement que l'usage des TIC en classe par les apprenants dans leurs activités d'apprentissage reste très limité, moins de $8 \%$ des répondants permettent à leurs élèves d'exploiter fréquemment les TIC en classe à des fins d'apprentissage. Le faible usage des TIC par les apprenants est dû probablement à plusieurs facteurs : Insuffisance d'équipements pédagogiques adéquats, degré de maitrise des TIC par les enseignants et les apprenants, surnombre des élèves en classe, approches pédagogiques adoptées par le système éducatif ...

\begin{tabular}{|c|c|c|}
\hline & $\mathrm{Nb}$ & \\
\hline Jamais & 64 & | $35,35 \%$ \\
\hline Rarement & 55 & $30,38 \%$ \\
\hline Occasionnellement & 49 & $27,07 \%$ \\
\hline Souvent & 13 & 7,18\% \\
\hline
\end{tabular}

Figure 9. Usage des TIC en classe à des fins d'apprentissage par les apprenants

De même, la Figure 10 révèle que l'usage des TIC pour maintenir la communication en dehors des activités en classes est également limité, seulement environ 13,8\% des répondants qui utilisent les TIC pour garder la communication de façon régulière avec les apprenants. Ce résultat peut être expliqué par la non maitrise de l'utilisation des systèmes de gestion des apprentissages en ligne ou par l'incertitude vis-à-vis des apports de la communication et de l'enseignement à distance. De ce fait, la formation de nos enseignants dans ce domaine nous semble obligatoire pour permettre à nos élèves d'apprendre plus et mieux grâce aux moyens de communication et de formation à distance surtout selon l'approche pédagogique de la classe inversée.

\begin{tabular}{|l|r|r|}
\hline & \multicolumn{1}{|l|}{$\mathrm{Nb}$} & \\
\hline Jamais & 65 & \\
\hline Rarement & 39 & \\
\hline Occasionnellement & 52 & \multicolumn{2}{|c|}{$21,5 \%$} \\
\hline Souvent & 25 & \\
\hline
\end{tabular}

Figure 10. Usage des TIC pour maintenir la communication en dehors des activités en classes 


\section{La Perception des Enseignants des Bénéfices Tirés de L'intégration Pédagogique des TIC en Sciences de la Vie et de la Terre}

Pour cet item, nous avons retenu 7 modalités qui représentent les principaux apports et bénéfices tirés de l'intégration pédagogique des TIC en sciences de la vie et de la terre. (cf. Tableau 2 ci-dessous). Les enseignants devraient répondre, à chaque modalité, à l'aide d'une échelle de Likert en quatre points variant de « tout à fait d'accord » à « pas d'accord du tout ». Le tableau suivant regroupe les réponses obtenues selon chaque modalité:

Tableau 2. Perception des enseignants des bénéfices tirés de l'intégration des TIC en SVT

\begin{tabular}{|c|c|c|c|c|}
\hline & $\begin{array}{c}\text { Tout à } \\
\text { fait } \\
\text { d'accord }\end{array}$ & $\begin{array}{c}\text { Plutôt } \\
\text { d'accord }\end{array}$ & $\begin{array}{c}\text { Plutôt } \\
\text { pas } \\
\text { d'accord }\end{array}$ & $\begin{array}{c}\text { Pas } \\
\text { d'accord } \\
\text { du tout }\end{array}$ \\
\hline La motivation et l'incitation à être plus actif & $76,4 \%$ & $21,3 \%$ & $2,3 \%$ & $0,0 \%$ \\
\hline L'autonomie dans l'apprentissage & $42,6 \%$ & $40,9 \%$ & $11,9 \%$ & $4,5 \%$ \\
\hline La richesse et la diversité des ressources & $77,4 \%$ & $20,3 \%$ & $2,3 \%$ & $0,0 \%$ \\
\hline La compréhension des phénomènes complexes & $82,5 \%$ & $15,2 \%$ & $1,7 \%$ & $0,6 \%$ \\
\hline L'assimilation et la mémorisation des & $59,6 \%$ & $31,3 \%$ & $6,3 \%$ & $2,8 \%$ \\
\hline connaissances & $45,4 \%$ & $37,9 \%$ & $10,9 \%$ & $5,7 \%$ \\
\hline L'auto-évaluation (exercices interactifs) & $56,8 \%$ & $31,8 \%$ & $9,1 \%$ & $2,3 \%$ \\
\hline L'amélioration des résultats scolaires & & & & \\
\hline
\end{tabular}

Ces résultats montrent que globalement, en moyenne, plus de $90 \%$ des enseignants sont d'accord (tout à fait d'accord et plutôt d'accord) avec l'ensemble des bénéfices tirés de l'intégration des TIC en sciences de la vie et de la terre. Les bénéfices qui ont reçu un jugement très favorable (plus de 97\%) relèvent de la motivation et l'incitation à être plus actif, de la richesse et la diversité des ressources, et de la compréhension des phénomènes complexes. Les autres apports tirés de l'usage des TIC dans l'enseignement et l'apprentissage, relevant de l'autonomie dans l'apprentissage, de l'assimilation et la mémorisation des connaissances, de l'auto-évaluation, et enfin de l'amélioration des résultats scolaires, ont aussi reçu un jugement favorable (d'accord) de $83 \%$ à $90 \%$ des enseignants.

Quant aux réfractaires au changement, ils représentent dans notre enquête moins de 9\%. Ces enseignants préfèrent voir les preuves de l'apport bénéfique des TIC avant de les utiliser eux-mêmes (Villeneuve, 2011). Nous pouvons donc à ce titre conclure que généralement les enseignants des SVT enquêtés sont très convaincus du rôle que peuvent jouer l'usage des TIC dans l'amélioration de la qualité de l'enseignement et d'apprentissage.

Le questionnement sur la perception des enseignants des bénéfices tirés de l'intégration pédagogique des TIC dans la pratique enseignante permet de révéler l'aptitude et la prédisposition des enseignants à s'investir pour une bonne intégration de ces outils en classe. En outre, certains chercheurs ont 
constaté que la clé de l'intégration pédagogique des technologies éducatives passe avant tout par des attitudes positives envers les TIC (Player-Koro, 2007). Enfin, la représentation positive des enseignants vis-à-vis de l'introduction des TIC dans l'environnement éducatif est certainement influencée par l'intégration progressive de la technologie dans la vie courante (Biaz, 2009).

\section{Les Obstacles à L'intégration des TIC en Classe}

Les résultats assemblés nous indiquent que la majorité des enseignants interrogés déclarent ne pas utiliser les TIC à cause de l'insuffisance des équipements dans leurs établissements $(77,9 \%)$, du surnombre des élèves en classe $(61,9 \%)$ ou du manque de soutien technique $(50,8 \%)$. D'autres enseignants (moins de $50 \%$ ) considèrent que ce sont plutôt le manque de temps, la formation insuffisante dans ce domaine ou les approches pédagogiques adoptées par le système éducatif qui entravent l'utilisation des TIC. Une minorité des enseignants (environ 11\%) déclare l'incertitude vis-àvis les apports des TIC dans l'enseignement comme étant un obstacle à l'intégration des TIC.

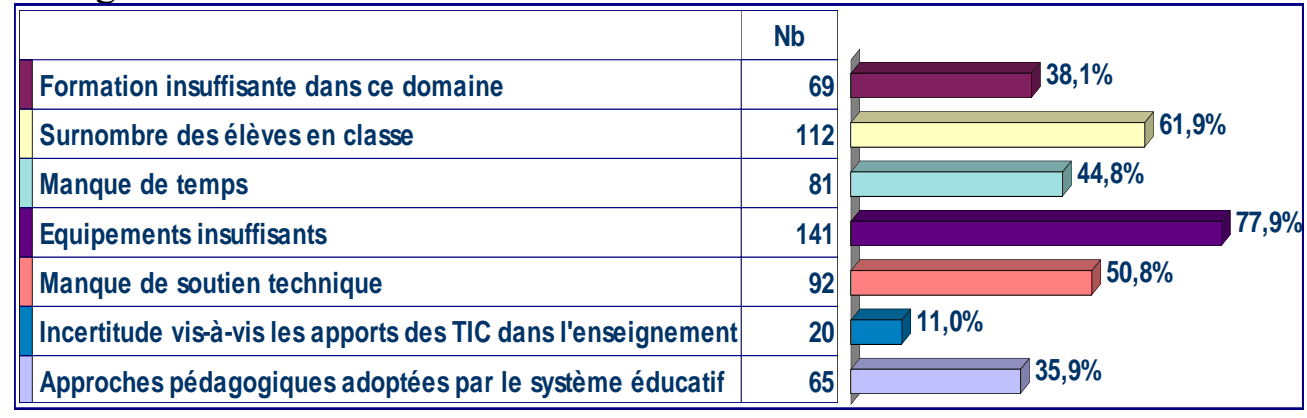

Figure 11. Les obstacles à l'intégration des TIC en classe

L'analyse quantitative des données recueillies nous a permis de mettre en relief les principaux obstacles à l'intégration des TIC dans l'enseignement des SVT au Maroc. En fait, trois catégories principales d'obstacles sont perçues par les enseignants.

- $\quad$ La première catégorie d'obstacles est celle relative à l'insuffisance de l'équipement technologique (tableau blanc interactif, connexion internet, matériels d'EXAO). Ce résultat concorde avec les résultats qui confirment que le manque ou l'insuffisance des équipements technologiques et la disponibilité de l'accès à Internet figurent parmi les contraintes considérables à la réussite de l'intégration des TIC en éducation (W. J. Pelgrum et al., 2004; Bibeau, 2005; Messaoudi et al., 2012; Unal et al., 2012; Gil-Flores et al., 2017).

La seconde catégorie d'obstacles, perçue par les enseignants concerne les problèmes liés au contexte éducatif marocain et particulièrement l'effectif élevé d'élèves par classe, ce problème rend difficile voire impossible d'amener les apprenants à utiliser les TIC dans des situations d'apprentissage 
et d'évaluation. D'autres contraintes qui sont liées aux conditions de travail, à la charge des contenus des programmes scolaires dans certains niveaux d'enseignement au secondaire, et aux approches pédagogiques adoptées par le système éducatif, pourraient freiner l'utilisation efficace des TIC dans l'enseignement (Mastafi, 2014).

- La troisième catégorie d'obstacles correspond à la formation en matière d'intégration des TIC et au soutien technique. La plupart des recherches réalisées à travers le monde ont conclu que le manque de formation des enseignants dans le domaine des TIC représente un obstacle majeur dissuadant de faire usage des technologies dans les pratiques d'enseignement (Pelgrum et al., 1999; Pelgrum et al., 2004; Balanskat et al., 2006; Taylor et al., 2007; Alwani et al., 2010; Buabeng-Andoh, 2012; Gil-Flores et al., 2017). De même l'absence du soutien technique permettant d'aider les enseignants à surmonter les difficultés qui accompagnent l'usage des TIC constitue un frein à l'usage des TIC dans les pratiques éducatives (Mastafi, 2014).

\section{Les Conditions D'intégration des TIC}

Les résultats de cet item viennent encore confirmer ce qui a été dit précédemment sur les écueils à l'intégration effective des TIC dans l'enseignement de la discipline des sciences de la vie et de la terre. En plus, les données de la Figure 12 montrent, selon la conception des questionnés, les conditions essentielles d'une intégration réussie des TIC en SVT au secondaire.

\begin{tabular}{|c|c|c|}
\hline & $\mathrm{Nb}$ & \\
\hline Suivre une formation en informatique & 59 & $32,6 \%$ \\
\hline Suivre une formation dans la conduite de l'enseignement intégrant les TIC & 113 & $62,4 \%$ \\
\hline Avoir des classes non chargées & 123 & $68,0 \%$ \\
\hline Avoir des équipements matériels suffisants & 157 & $86,7 \%$ \\
\hline Avoir des logiciels éducatifs convenables & 136 & $75,1 \%$ \\
\hline Autre & 21 & $11,6 \%$ \\
\hline
\end{tabular}

Figure 12. Les conditions d'intégration des TIC

Les conditions les plus importantes indiquées par les répondants de notre enquête sont essentiellement:

- $\quad$ Les équipements matériels doivent être suffisants (pour plus de 86,7\% des enseignants) et la disponibilité des logiciels éducatifs convenables (pour trois quarts des répondants). Sachant que, l'équipement en TIC joue un rôle fondamental dans leur utilisation en classe, si les acteurs pédagogiques ne disposent pas d'un accès facilité à un matériel adéquat, il est probable qu'ils seront moins prédisposés à faire l'effort d'utiliser les TIC avec leurs élèves (Heer et al., 2006). 
- L'effectif d'élèves par classe doit être réduit selon plus de deux tiers des répondants pour encourager l'usage des TIC. Ce résultat est en concordance avec ceux de l'enquête menée par Alluin et ses collaborateurs (2010), considérant que le nombre d'élèves est le principal facteur qui peut dissuader les enseignants de l'usage des TIC. En effet, l'augmentation des effectifs d'élèves combinée avec d'autres facteurs comme l'insuffisance des équipements peut véritablement entraver l'utilisation optimale des TIC à des fins pédagogiques (Ngono, 2012). Pour cela, le ministère de l'éducation nationale doit adopter en urgence des mesures appropriées afin d'atténuer l'impact négatif des effectifs trop élevés sur l'usage des TIC et aussi bien sur l'encadrement pédagogique des élèves au Maroc.

- La formation dans la conduite de l'enseignement intégrant les TIC s'impose vraiment selon environ les deux tiers des répondants comme une condition d'intégration réussie et efficiente des TIC dans l'enseignement. La formation dans ce domaine peut jouer un rôle primordial en ce qui concerne la réduction du facteur d'incertitude et, par conséquent, elle favorise une meilleure approbation de l'innovation induite par les TIC (Carugati et al., 2002; Heer et al., 2006). D'autres travaux de recherche ont mentionné que la qualification technique des enseignants praticiens leur confère la confiance face aux outils technologiques qui est une condition nécessaire pour l'estime de leur propre performance face à l'exploitation de la technologie (Carugati et al., 2002; Leu, 2005).

Ainsi, il est important de préciser qu'une intégration efficace des TIC à des fins pédagogiques ne peut se réaliser sans formation (Heer et al., 2006; Aoki, 2013), et que cette dernière ne s'apprend pas immédiatement lors d'une courte formation, c'est un processus continu qui nécessite temps et effort (Sheingold et al., 1990). Définitivement, pour une implémentation réussie des TIC dans les pratiques éducatives, les enseignants doivent avoir les connaissances de base en matière d'utilisation et d'exploitation des logiciels éducatifs (Biaz, 2009) et doivent développer leurs compétences didactiques et pédagogiques liées aux technologies (Guir, 2002; Bingimlas, 2009; Kirkland et al., 2009; Koh et al., 2014; Lee et al., 2014; Valtonen et al., 2015)

Finalement, en plus des conditions fondamentales précitées, il y a d'autres facteurs qui peuvent entre autres encourager l'utilisation des TIC dans l'éducation : la motivation des acteurs pédagogiques pour déployer plus d'efforts, la volonté d'améliorer les résultats scolaires des élèves, le plaisir de fournir aux élèves des documents de haute qualité, le souci de renforcer l'autonomie des élèves et enfin les recommandations institutionnelles (Alluin et al., 2010; Aoki et al., 2013). 


\section{Conclusion et Perspectives}

La présente étude visait à présenter un diagnostic de la réalité de l'usage des TIC dans l'enseignement des SVT au secondaire dans le contexte éducatif marocain, à identifier les perceptions des enseignants des SVT des bénéfices véritablement apportés par 1'intégration pédagogique de TIC dans l'éducation et finalement à déterminer, selon la conception des enseignants eux-mêmes, les principaux facteurs qui entravent ou facilitent une intégration effective et efficiente des TIC dans la pratique enseignante. Pour ce faire, une enquête a été menée auprès de 181 enseignants des sciences de la vie et de la terre répartis sur un nombre important des établissements scolaires (collèges et lycées).

Nos résultats montrent que l'utilisation des TIC dans l'enseignement des SVT en classe et hors classe est limitée. De même l'usage des TIC par les élèves à des fins d'apprentissage reste encore très faible malgré que la majorité des enseignants interrogés sont très convaincus de la richesse des opportunités pédagogiques induites par l'intégration des TIC dans les pratiques pédagogiques.

D'autre part, notre enquête nous a permis d'identifier les principaux facteurs entravant l'intégration réussie et raisonnée des TIC dans l'enseignement. Les enseignants répondants ont déclaré que les principaux obstacles qui peuvent les dissuader de l'usage des TIC sont en premier lieu des obstacles liés à l'insuffisance des équipements, puis les contraintes liées au contexte éducatif marocain et particulièrement l'enseignement dans une classe à large effectif, et enfin la problématique de la formation en matière d'intégration des TIC et le manque de soutien technique.

Symétriquement aux facteurs qui peuvent dissuader, il existe des facteurs qui peuvent encourager les enseignants de faire usage des TIC. En effet, avoir des équipements matériels suffisants, avoir des logiciels éducatifs convenables, avoir des classes non chargées et suivre une formation dans la conduite de l'enseignement intégrant les TIC sont les facteurs les plus encourageants à l'usage des TIC selon la perception des enseignants enquêtés.

Ainsi, les projets de généralisation des TIC dans le système éducatif marocain devraient prendre en considération les principales contraintes soulignées dans cette recherche et les recherches précédentes menées dans notre pays afin d'intégrer sur des bases plus solides l'ère numérique dans nos établissements scolaires.

Enfin, il nous semble très pertinent de poursuivre des recherches dans ce domaine pour comprendre comment aider les enseignants praticiens à progresser vers une intégration optimale et harmonieuse des TIC et pour mieux connaitre ces attitudes auprès des directeurs des établissements scolaires, des inspecteurs pédagogiques et des élèves. 


\section{References:}

1. Abouhanifa, S., Kabbaj, M., Belmadani, M., Khalfaoui, M. \& Hanini, M. (2008). L'outil informatique : Défis d'intégration et objet de formation des enseignants de mathématiques dans le secondaire. Revue Sesamath $\mathrm{n}^{\circ} 8$.

2. Abouzaid, A., Taoufik, M., Moufti, A., \& Lamsalmi, A. (2017). Intégration des TICE dans l'enseignement des Sciences de l'Ingénieur dans la filière Sciences Technologies Mécaniques aux lycées du Maroc: Réalité et Obstacles. European Scientific Journal, ESJ, 13(13).

3. Agence Nationale de Réglementation des Télécommunications (2017). Résultats de l'enquête annuelle de collecte des indicateurs TIC auprès des ménages et des individus Année 2016. https://www.anrt.ma/sites/default/files/publications/enquete_tic_2016 _fr.pdf

4. Ahaji, K., El Hajjami, A., Ajana, L., El Mokri, A., \& Chikhaoui, A. (2008). Analyse de l'effet d'intégration d'un logiciel d'optique géométrique sur l'apprentissage d'élèves de niveau baccalauréat sciences expérimentales. Revue EpiNet $\mathrm{n}^{\circ} 101$.

5. Ahaji, K., Zahim, S., \& Badda, B. (2012). Soubassements théoriques pour guider l'intégration réussie des TICE. EpNet: Revue Electronique De l'EPI, 142.

6. Ait kaikai, H. (2014). Appropriation des technologies de l'information et de la communication au sein de l'université marocaine : perceptions des étudiants : ownership of information technology and communication in the moroccan university: student's perceptions. Frantice.net, numéro 8

7. Alj, O. \& Benjelloun, N. (2016). Impact de l'utilisation du Logiciel d'Algorithme et de Résolution de Problème (LARP) dans le processus enseignement-apprentissage de l'algorithmique au secondaire qualifiant : The Impact of the use of Algorithm and Problem Solving Software (LARP) on the teaching-learning process of algorithms in High School. frantice.net, Numéro 11 - Décembre 2015

8. Alj, O. \& Benjelloun, N. (2013). Intégration des TIC dans l'enseignement des sciences physiques au Maroc dans le cadre du programme GENIE : difficultés et obstacles. Revue internationale des technologies en pédagogie universitaire (RITPU), 10(2), 49-65.

9. Alluin, F., Billet, M., \& Gentil, R. (2010). Les technologies de l'information et de la communication (TIC) en classe au collège et au lycée : éléments d'usages et enjeux. Ministère de l'Education nationale, Direction de l'évaluation, de la prospective et de la performance. 
10. Alwani, A. E. S. \& Soomro, S. (2010). Barriers to effective use of information technology in science education at Yanbu Kingdom of Saudi Arabia. In E-learning experiences and future. InTech.

11. Aoki, H., Kim, J., \& Lee, W. (2013). Propagation \& level: Factors influencing in the ICT composite index at the school level. Computers \& Education, 60(1), 310-324.

12. Balanskat, A., Blamire, R., \& Kefala, S. (2006). The ICT impact report. European Schoolnet, 1, 1-71.

13. Basque, J. \& Lundgren-Cayrol, K. (2003). Une typologie des typologies des usages des «TIC» en éducation. Télé-Université, Québec.

14. Benfares, S., Alami, A., Moncef, Z., Chikhaou, A., \& Elazami, M. (2015). Integration of ict in environmental education - case study on the greenhouse effect among secondary School students. International Journal of Research in Education Methodology, 7(2), 1077-1087.

15. Benfaress, S., Zaki, M., \& Alami, A. (2016). Analyse Multidimensionnelle Des Facteurs D’intégration Des Tice: Etude De Cas À Travers Le Programme Genie Marocain. European Scientific Journal, ESJ, 12(19).

16. Biaz, A., Bennamara, A., Khyati, A., \& Talbi, M. (2009). Intégration des technologies de l'information et de la communication dans le travail enseignant, état des lieux et perspectives. EpiNet: revue électronique de l'EPI, 120.

17. Bibeau, R. (2005). Les TIC à l'école : proposition de taxonomie et analyse des obstacles à leur intégration. Revue EpiNet $\mathrm{n}^{\circ} 79$

18. Bingimlas, K. A. (2009). Barriers to the successful integration of ICT in teaching and learning environments: A review of the literature. Eurasia Journal of Mathematics, Science \& Technology Education, $5(3)$.

19. Boticki, I., Baksa, J., Seow, P., \& Looi, C. K. (2015). Usage of a mobile social learning platform with virtual badges in a primary school. Computers \& Education, 86, 120-136.

20. Buabeng-Andoh, C. (2012). Factors influencing teachers' adoption and integration of information and communication technology into teaching: A review of the literature. International Journal of Education and Development using Information and Communication Technology, 8(1), 136.

21. Carugati, F. \& Tomasetto, C. (2002). Le corps enseignant face aux technologies de l'information et de la communication: un défi incontournable. Revue des sciences de l'éducation, 28(2), 305-324. 
22. Collin, S. \& Karsenti, T. (2013). Usages des technologies en éducation: analyse des enjeux socioculturels. Éducation et francophonie, 41(1), 192-210.

23. CSEFR. (2015). Vision stratégique de la réforme 2015-2030. Pour une école de l'équité, de la qualité et de la promotion. Http://www.csefrs.ma/pdf/vision_vf_fr.pdf

24. Depover, C., Karsenti, T., \& Komis, V. (2007). Enseigner avec les technologies : favoriser les apprentissages, développer des compétences. PUQ.

25. Droui, M., El Hajjami, A., Bouklah, M., \& Zouirech, S. (2013). Impact de l'apprentissage par problème sur la compréhension conceptuelle de la mécanique newtonienne. EpiNet : Revue électronique de l'EPI, 157

26. El hassouny, E., Kaddari, F., Elachqar, A., \& Alami, A. (2014). Teaching/Learning Mechanics in High School with the Help of Dynamic Software. Procedia-Social and Behavioral Sciences, 116, 4617-4621.

27. El Madhi, Y., Chiahou, B., Belghyti, D., El Kharrim, K., \& El Halouani, H. (2014). LES contraintes liées à l'intégration du TIC dans l'enseignement des sciences de la vie et de la terre au Maroc. European Scientific Journal, ESJ, 10(34).

28. El Ouidadi, O., Lakdim, A., Essafi, K., \& Sendide K. (2011). Contribution à l'évaluation de l'impact de l'intégration des TICE dans l'enseignement et l'apprentissage : exemple de la division cellulaire (mitose) en deuxième année de baccalauréat sciences expérimentales. EpiNet $\mathrm{n}^{\circ} 138$.

29. El Ouidadi, O., Essafi, K., Aboutajdyne, M., Sendide, K., \& Depiereux, E. (2013). Principaux facteurs influençant les usages des TICE chez des enseignants marocains. frantice.net, numéro 6

30. Forget, D. (2005). Impacts des TIC dans l'enseignement collégial : une métasynthèse réalisée par l'association pour la recherche au collégial. Revue Pédagogie collégiale, 18(3), 43-47.

31. Fortin, M. F., Côté, J., \& Filion, F. (2006). Fondements et étapes du processus de recherche (p. 485). Montréal: Chenelière éducation.

32. Gil-Flores, J., Rodríguez-Santero, J., \& Torres-Gordillo, J. J. (2017). Factors that explain the use of ICT in secondary-education classrooms: The role of teacher characteristics and school infrastructure. Computers in Human Behavior, 68, 441-449.

33. Guennoun, B. \& Benjelloun, N. (2016). Regards des étudiants sur l'intégration des TIC dans l'enseignement supérieur scientifique. Revue internationale des technologies en pédagogie universitaire/International Journal of Technologies in Higher Education, 13(1), 64-94. 
34. Guir, R. (2002). Pratiquer les TICE: former les enseignants et les formateurs à de nouveaux usages. De Boeck Supérieur.

35. Heer, S. \& Akkari, A. (2006). Intégration des TIC par les enseignants: premiers résultats d'une enquête suisse. Revue internationale des technologies en pédagogie universitaire, 3(3), 38-48.

36. Huot, D., France, H., \& Parks, S. (2009). Les technologies de l'information et de la communication (TIC) à l'école secondaire: une étude longitudinale. Presses de l'Université Laval.

37. Karsenti, T. (2003). Favoriser la motivation et la réussite en contexte scolaire: Les TIC feront-elles mouche. Vie pédagogique, 127, 27-32.

38. Karsenti, T. \& Larose, F. (2001). Les TIC... au cœur des pédagogies universitaires. Québec : Presses de 1'Université du Québec.

39. Kirkland, K. \& Sutch, D. (2009). Overcoming the barriers to educational innovation. A literature review. In FutureLab (Ed.). Bristol.

40. Koh, J. H. L., \& Chai, C. S. (2014). Teacher clusters and their perceptions of technological pedagogical content knowledge (TPACK) development through ICT lesson design. Computers \& Education, 70, 222-232.

41. Laaziz, I. (2016). Programme génie promotion de l'usage des logiciels \& ressources éducatives libres. Open education day morocco oer strategy forum cadi ayyad university, Marrakech 6-7 décembre.

42. Lakdim, A., El Ouidadi, O., Essafi, K., \& Sendide, K. (2012). Etude de l'impact de l'utilisation d'une plate-forme en ligne pour la régulation des apprentissages et l'accompagnement des élèves en SVT : Cas des lycées de la ville de Fès (Maroc). EpiNet $\mathrm{n}^{\circ} 144$.

43. Lebrun, M. (2011). Impacts des TIC sur la qualité des apprentissages des étudiants et le développement professionnel des enseignants : vers une approche systémique. Sciences et Technologies de l'Information et de la Communication pour l'Éducation et la Formation (STICEF), 18, 20-pages.

44. Lee, Y. \& Lee, J. (2014). Enhancing pre-service teachers' self-efficacy beliefs for technology integration through lesson planning practice. Computers \& Education, 73, 121-128.

45. Leu, E. (2005). The Role of Teachers, Schools, and Communities in Quality Education: A Review of the Literature. Academy for Educational Development.

46. Maddux, C. D., \& Johnson, D. L. (2012). External validity and research in information technology in education. Computers in the schools, 29(3), 249-252. 
47. Maouni, A., Mimet, M., khaddor, M., Madrane, M., \& Moumene, M. (2014). L'intégration des TIC dans l'enseignement des SVT au Maroc : réalité et attentes. RADISMA, Numéro 10.

48. Mastafi, M. (2013). Intégration et usages des TIC dans le système éducatif marocain: Attitudes des enseignants de l'enseignement primaire et secondaire. Adjectif.net

49. Mastafi, M. (2014). Obstacles à l'intégration des technologies de l'information et de la communication (TIC) dans le système éducatif marocain : Barriers to integration of information and communication technology (ICT) in Moroccan education system. frantice.net, Numéro 8.

50. Messaoudi, F. \& Talbi, M. (2012). Réussir l'intégration des TICE au Maroc : regard sur le déploiement de la stratégie nationale GENIE. Revue EpiNet n ${ }^{\circ} 143$

51. Monsakul, J. (2008). A research synthesis of instructional technology in higher education. In Society for Information Technology \& Teacher Education International Conference (pp. 2134-2139). Association for the Advancement of Computing in Education (AACE).

52. Nachit, B., Lablidi, A., Abdelwahed, N., Bahra, M., \& Talbi, M. (2013). Concours des enseignants innovants au Maroc. EpiNet: Revue Electronique de l'Epi, 157.

53. Nafidi, Y., Alami, A., Zaki, M., \& Afkar, H. (2015). Open and distance learning in the initial education of trainee teachers. International Journal of Education Learning and Development, 3(9), 53-64.

54. Nafidi, Y., Alami, A., \& Zaki, M. (2015). Impacts de l'usage d'une simulation numérique sur l'apprentissage en sciences de la terre. 1ère Edition du Workshop International sur les Approches Pédagogiques \& E-Learning.

55. Ngono, M. (2012). Intégration des Technologies de l'Information et de la Communication dans l'enseignement secondaire au Cameroun : Point de vue des enseignantes et enseignants des collèges et lycées de la ville de Yaoundé. Thèse de Doctorat. Faculté des Sciences de l'Education, Université Laval.

56. Ouazzani Touhami, A., Benjelloun, N., Aami, M., \& Haddou, A. (2014). Impact of using Java Software of Geometrical Optics JSGO on the construction success of the virtual image among the first year university students. IOSR Journal of Research \& Method in Education (IOSR-JRME), Volume 4, Issue 3 Ver II, pp. 16-24.

57. Ou-Zennou, F., El Mahmoudi, A., \& Bouderhem, N. (2016) : TICE au service de l'évaluation sommative des apprentissages dans les établissements scolaires marocains. EpiNet: revue electronique de l'epi, 186. 
58. Pelgrum, W. J. \& Anderson, R. E. (Eds.). (1999). ICT and the emerging paradigm for life long learning: A worldwide educational assessment of infrastructure, goals and practices. International Association for the Evaluation of Educational Achievement.

59. Pelgrum, W. J. \& Law, N. (2004). Les TIC et l'éducation dans le monde : tendances, enjeux et perspectives. UNESCO, Institut international de planification de l'éducation.

60. Peraya, D. (2002). Communication et nouvelles technologies. In p. Perrig-chiello et f. Darbellay (dir.), qu'est-ce que l'interdisciplinarité ? Les nouveaux défis de l'enseignement (p.117-143). Lausanne : institut universitaire kurt Bösch

61. Peraya, D. (2005). La formation à distance : un dispositif de formation et de communication médiatisées. Une approche des processus de médiatisation et de médiation. Technologies Développement Recherche, (0a).

62. Peraya, D. (2014). Distances, absence, proximités et présences : des concepts en déplacement. Distances et médiations des savoirs. Distance and Mediation of Knowledge, 2(8).

63. Peraya, D., Viens, J., \& Karsenti, T. (2002). Introduction : Formation des enseignants à l'intégration pédagogique des TIC: Esquisse historique des fondements, des recherches et des pratiques. Revue des sciences de l'éducation, 28(2), 243-264.

64. Perreault, N. (2003). Rôle et impact des TIC sur l'enseignement et l'apprentissage au collégial-I. Pédagogie collégiale, 16(3), 3-10.

65. Perrenoud, P. (1998). Se servir des technologies nouvelles. Genève : Faculté de psychologie et de sciences de l'éducation, Université de Genève.

66. Player-Koro, C. (2007). Why teachers make use of ICT in education. In Arbetspaper presenterat vid 10th Pre-Conference of Junior Researchers of Earli, 27-28 augusti, Budapest, Ungern.

67. Poyet, F. \& Drechsler, M. (2009). Impact des TIC dans l'enseignement: une alternative pour l'individualisation. Dossier d'actualité de la VST, 41.

68. Raby, C., Karsenti, T., Meunier, H., \& Villeneuve, S. (2011). Usage des TIC en pédagogie universitaire: point de vue des étudiants. Revue internationale des technologies en pédagogie universitaire/International Journal of Technologies in Higher Education, 8(3), 6-19.

69. Redecker, C., Ala-Mutka, K., Bacigalupo, M., Ferrari, A. \& Punie, Y. (2009). Learning 2.0: the impact of web 2.0 innovations on education and training in Europe. Séville, Espagne : Institute for Prospective Technological Studies. 
70. Rogers, G. (2004). History, learning technology and student achievement: making the difference?. Active Learning in Higher Education, 5(3), 232-247.

71. Royaume du Maroc (1999). Projet de charte nationale d'éducation et de formation. Commission spéciale éducation-formation.

72. Saunders, G. \& Klemming, F. (2003). Integrating technology into a traditional learning environment: Reasons for and risks of success. Active learning in higher education, 4(1), 74-86.

73. Sheingold, K. \& Hadley, M. (1990). Accomplished teachers: Integrating computers into classroom practice. New York: Center for Technology in Education, Bank Street College of Education.

74. Szabo, Z. \& Schwartz, J. (2009, June). Learning methods for teacher education: Blackboard discussions produce deep learning. In EdMedia: World Conference on Educational Media and Technology (pp. 2323-2334). Association for the Advancement of Computing in Education (AACE).

75. Taylor, N. \& Corrigan, G. (2007). New South Wales Primary School Teachers' Perceptions of the Role of ICT in the Primary Science Curriculum--A Rural and Regional Perspective. International Journal of Science and Mathematics Education, 5(1), 85-109.

76. Traoré, D. (2008). Quel avenir pour l'usage pédagogique des TIC en Afrique subsaharienne? Cas de cinq pays membres du ROCARE. ICT and changing mindsets in Education. Bamenda, Cameroun: Langaa.

77. Turney, C. S. M., Robinson, D., Lee, M., \& Soutar, A. (2009). Using technology to direct learning in higher education: The way forward? Active Learning in Higher Education, 10(1), 71-83.

78. Unal, S. \& Ozturk, I. H. (2012). Barriers to ITC integration into teachers' classroom practices: Lessons from a case study on social studies teachers in Turkey. World Applied Sciences Journal, 18(7), 939-944.

79. Valtonen, T., Kukkonen, J., Kontkanen, S., Sormunen, K., Dillon, P., \& Sointu, E. (2015). The impact of authentic learning experiences with ICT on pre-service teachers' intentions to use ICT for teaching and learning. Computers \& Education, 81, 49-58.

80. Villeneuve, S. (2011). L'évaluation de la compétence professionnelle des futurs maitres du Québec à intégrer les technologies de l'information et des communications (TIC) : maitrise et usages. Thèse de doctorat inédite. Montréal : Université de Montréal.

81. Yang, X., Li, X., \& Lu, T. (2015). Using mobile phones in college classroom settings: Effects of presentation mode and interest on concentration and achievement. Computers \& Education, 88, 292-302. 